\title{
Soft corrections to inclusive DIS at four loops and beyond
}

\section{Goutam Das*}

Theory Group, Deutsches Elektronen-Synchrotron (DESY), D-22607 Hamburg, Germany

E-mail: goutam.das@desy.de

\section{Sven-Olaf Moch}

II. Institute for Theoretical Physics, Hamburg University, D-22761 Hamburg, Germany

E-mail: sven-olaf.mochedesy.de

\section{Andreas Vogt}

Department of Mathematical Sciences, University of Liverpool, Liverpool L69 3BX, United Kingdom E-mail: andreas.vogteliverpool.ac.uk

We study the threshold corrections to the structure functions in deep-inelastic scattering (DIS) at the fifth logarithmic $\left(\mathrm{N}^{4} \mathrm{LL}\right)$ order of the soft-gluon exponentiation in massless perturbative QCD. Using recent results for the splitting functions and the quark form factor, we derive the fourthorder contribution to the coefficient $f^{\mathrm{q}}$ of the form factor and from it the $\mathrm{N}^{4} \mathrm{LL}$ part of the exponentiation coefficient $B^{\text {DIS }}$ in the limit of a large number of colours. An approximation scheme is shown that leads to sufficiently accurate $\mathrm{N}^{4} \mathrm{LL}$ results for full QCD. The $\mathrm{N}^{4} \mathrm{LL}$ corrections are small and lead to a further stabilization of the perturbative expansion for the soft-gluon exponent.

XXVII International Workshop on Deep-Inelastic Scattering and Related Subjects - DIS2019 8-12 April, 2019, Torino, Italy

\footnotetext{
* Speaker.
} 


\section{Introduction}

The Wilson coefficients (coefficient functions) for the structure functions of inclusive DIS have been a subject of research since the early days of QCD. These quantities are not only relevant for determining the parton distribution functions (PDFs) and the strong coupling constant $\alpha_{\mathrm{s}}$ using structure function data, see, e.g., [1], but also to other processes and less inclusive observables in DIS, see, e.g., [2]. The main Wilson coefficients for DIS are presently known up to the third order in $\alpha_{\mathrm{s}}$ in massless perturbative QCD [3]. Their perturbative expansion is well-behaved except close to the kinematic endpoints $x=0$ and $x=1$ of the Bjorken variable. The dominant terms $\ln ^{\ell}(1-x) /(1-x)_{+}$in the latter (threshold) limit are resummed by the soft-gluon exponentiation, see, e.g., [4-6], which is best formulated in Mellin $N$-space [4]. So far this resummation has been performed up to the next-to-next-to-next-to-leading logarithmic $\left(\mathrm{N}^{3} \mathrm{LL}\right)$ accuracy [7].

The threshold resummation coefficients are closely related to the large- $x$ limit of the quarkquark splitting functions for the PDFs and to the quark form factor $[8,9]$ which are both fully known to order $\alpha_{\mathrm{s}}^{3}$ [10-13]. Recently the computations of these quantities have been extended to order $\alpha_{\mathrm{s}}^{4}$ in the $\left(\mathrm{L} n_{c}\right)$ limit of a large number of colours $[14,15]$. Together with approximate results for the $n_{f}$-independent [16] and exact expression for the $n_{f}$-dependent contributions to the cusp anomalous dimension in full QCD [17] these results facilitate the effective extension of the threshold resummation for the DIS Wilson coefficients to the next $\left(\mathrm{N}^{4} \mathrm{LL}\right)$ logarithmic order. In the following we recall the theoretical framework, present the $\mathrm{N}^{4} \mathrm{LL}$ resummation coefficient and briefly address the numerical implications of this result for the resummation of DIS in QCD.

\section{Theoretical framework and new fourth-order coefficients}

The all-order large- $N$ behaviour of the DIS Wilson coefficients for $F_{1}, F_{2}$ and $F_{3}$ can be written as

$$
C^{N}\left(Q^{2}\right)=g_{0}\left(Q^{2}\right) \cdot \exp \left[G^{N}\left(Q^{2}\right)\right]+\mathscr{O}\left(N^{-1} \ln ^{n} N\right),
$$

where the resummation exponent $G^{N}$ of the dominant $N^{0} \ln ^{n} N$ contributions is given by [18]

$$
G^{N}=\int_{0}^{1} d z \frac{z^{N-1}-1}{1-z}\left[\int_{\mu_{f}^{2}}^{(1-z) Q^{2}} \frac{d q^{2}}{q^{2}} A^{\mathrm{q}}\left(\alpha_{\mathrm{s}}\left(q^{2}\right)\right)+B^{\mathrm{DIS}}\left(\alpha_{\mathrm{s}}\left((1-z) q^{2}\right)\right)\right] .
$$

Here $A^{\mathrm{q}}$ is the (light-like) quark cusp anomalous dimension and $B^{\mathrm{DIS}}$ is the resummation coefficient for DIS. Both have perturbative series, $A^{\mathrm{q}}=\sum_{i} a_{s}^{i} A_{i}^{\mathrm{q}}$ etc, in terms of the strong coupling which we normalize as $a_{\mathrm{s}} \equiv \alpha_{\mathrm{s}} / 4 \pi$. Performing the integrations one can organize the exponent as

$$
G^{N}=\ln N g^{(1)}(\lambda)+g^{(2)}(\lambda)+a_{\mathrm{s}} g^{(3)}(\lambda)+a_{\mathrm{s}}^{2} g^{(4)}(\lambda)+a_{\mathrm{s}}^{3} g^{(5)}(\lambda)+\ldots,
$$

where $\lambda=\beta_{0} a_{\mathrm{s}} \ln N$ or $\lambda=\beta_{0} a_{\mathrm{s}} \ln \widetilde{N}$ with $\ln \widetilde{N}=\ln N+\gamma_{\mathrm{e}}$. The first $n+1$ terms in (2.3) are required for the resummation at $\mathrm{N}^{n} \mathrm{LL}$ accuracy. The $\mathrm{N}^{2} \mathrm{LL}$ and $\mathrm{N}^{3} \mathrm{LL}$ contributions to $G^{N}$ have been derived in [7, 19]; explicit expressions can be found in (3.3) - (3.6) of [7]. The lengthy new function $g^{(5)}(\lambda)$ entering at $\mathrm{N}^{4} \mathrm{LL}$ will be presented in [20]. The $N$-independent prefactor $g_{0}$ is presently known to order $\alpha_{\mathrm{s}}^{3}$ from the all- $N$ calculation in [3], see (4.6) - (4.8) of [7],

$$
g_{0}=1+a_{\mathrm{s}} g_{01}+a_{\mathrm{s}}^{2} g_{02}+a_{\mathrm{s}}^{3} g_{03}+\mathscr{O}\left(a_{\mathrm{s}}^{4}\right) .
$$


The resummation to $\mathrm{N}^{4} \mathrm{LL}$ requires the terms up to $A_{5}^{\mathrm{q}}$ and $B_{4}^{\mathrm{DIS}}$ in their corresponding expansions. The impact of the former quantity, for which a first estimate has been obtained in [21], is very small. $B^{\text {DIS }}$ can be calculated from knowledge of the quark form factor or the DIS Wilson coefficients. The form factor satisfies a differential equation which follows from the renormalization group and gauge invariance. Its solution can be found in terms of the cusp anomalous dimension $A^{\mathrm{q}}$ and the function $G^{\mathrm{q}}$ containing the quantity $f^{\mathrm{q}}$ related to a universal eikonal anomalous dimension and the coefficient $B^{\mathrm{q}}$ of $\delta(1-x)$ in the quark-quark splitting function. The four-loop coefficient of $G^{\mathrm{q}}$ (which appears in the $1 / \varepsilon$ coefficient in the solution of the form factor) can be written as

$$
G_{4}^{\mathrm{q}}=2 B_{4}^{\mathrm{q}}+f_{4}^{\mathrm{q}}+\beta_{2} f_{01}^{\mathrm{q}}+\beta_{1} f_{02}^{\mathrm{q}}+\beta_{0} f_{03}^{\mathrm{q}}+\mathscr{O}(\varepsilon),
$$

where the quantities $f_{0 \text { n }}^{\mathrm{q}}$ are (combinations of) known lower-order coefficients of $G^{\mathrm{q}}$, see [12] and (20) of [9]. Hence $f_{4}^{\mathrm{q}}$ can be determined in the large- $n_{c}$ limit from the results of $[14,15]$. We find

$$
\begin{aligned}
\left.f_{4}^{\mathrm{q}}\right|_{\mathrm{L} n_{c}}= & C_{F} n_{c}^{3}\left(\frac{9364079}{6561}-\frac{1186735}{729} \zeta_{2}-\frac{837988}{243} \zeta_{3}+\frac{115801}{27} \zeta_{4}+\frac{11896}{9} \zeta_{2} \zeta_{3}+3952 \zeta_{5}\right. \\
& \left.-\frac{4796}{9} \zeta_{3}^{2}-\frac{129547}{54} \zeta_{6}-416 \zeta_{2} \zeta_{5}-720 \zeta_{3} \zeta_{4}-1700 \zeta_{7}\right)+C_{F} n_{c}^{2} n_{f}\left(-\frac{247315}{432}\right. \\
& \left.+\frac{412232}{729} \zeta_{2}+\frac{102205}{243} \zeta_{3}-\frac{7589}{6} \zeta_{4}-\frac{824}{9} \zeta_{2} \zeta_{3}-\frac{740}{9} \zeta_{5}+\frac{2816}{9} \zeta_{3}^{2}+\frac{15611}{27} \zeta_{6}\right) \\
+ & C_{F} n_{c} n_{f}^{2}\left(\frac{329069}{17496}-\frac{22447}{729} \zeta_{2}+\frac{25300}{243} \zeta_{3}+\frac{140}{3} \zeta_{4}-\frac{176}{9} \zeta_{2} \zeta_{3}-\frac{856}{9} \zeta_{5}\right) \\
+ & C_{F} n_{f}^{3}\left(-\frac{16160}{6561}-\frac{16}{81} \zeta_{2}-\frac{400}{243} \zeta_{3}+\frac{128}{27} \zeta_{4}\right) .
\end{aligned}
$$

The $a_{\mathrm{s}}^{4}$ contribution to resummation $B^{\mathrm{DIS}}$ reads, in terms of the genuine four-loop contributions $f_{4}^{\mathrm{q}}$ and $B_{4}^{\mathrm{q}}$, which are exactly known only in the $\mathrm{L} n_{c}$ limit for now, and lower-order coefficients,

$$
\begin{aligned}
B_{4}^{\mathrm{DIS}} & =-f_{4}^{\mathrm{q}}-B_{4}^{\mathrm{q}}-\beta_{2}\left(f_{01}^{\mathrm{q}}+g_{01}-\frac{1}{2} \zeta_{2} A_{1}^{\mathrm{q}}\right)+\beta_{0}^{3}\left(3 \zeta_{2} f_{01}^{\mathrm{q}}+3 \zeta_{2} g_{01}+2 \zeta_{3} f_{1}^{\mathrm{q}}+2 \zeta_{3} B_{1}^{\mathrm{q}}\right. \\
& \left.+\frac{3}{2} \zeta_{4} A_{1}^{\mathrm{q}}-\frac{3}{4} \zeta_{2}^{2} A_{1}^{\mathrm{q}}\right)+\beta_{0} \beta_{1}\left(\frac{5}{2} \zeta_{2} f_{1}^{\mathrm{q}}+\frac{5}{2} \zeta_{2} B_{1}^{\mathrm{q}}+\frac{5}{3} \zeta_{3} A_{1}^{\mathrm{q}}\right)+\beta_{0}^{2}\left(3 \zeta_{2} f_{2}^{\mathrm{q}}+3 \zeta_{2} B_{2}^{\mathrm{q}}+2 \zeta_{3} A_{2}^{\mathrm{q}}\right) \\
& -\beta_{1}\left(f_{02}^{\mathrm{q}}+2 g_{02}-\left(g_{01}\right)^{2}-\zeta_{2} A_{2}^{\mathrm{q}}\right)-\beta_{0}\left(f_{03}^{\mathrm{q}}+3 g_{03}-3 g_{02} g_{01}-\left(g_{01}\right)^{3}-\frac{3}{2} \zeta_{2} A_{3}^{\mathrm{q}}\right), \quad(2.7)
\end{aligned}
$$

where $g_{0 i}$ are to be taken without the $\gamma_{\mathrm{e}}$ terms in (4.6) - (4.8) of [7]. Its explicit form is given by

$$
\begin{aligned}
\left.B_{4}^{\mathrm{DIS}}\right|_{\mathrm{L} n_{c}}= & C_{F} n_{c}^{3}\left(-\frac{2040092429}{139968}+\frac{23011973}{1944} \zeta_{2}+\frac{517537}{36} \zeta_{3}-\frac{312481}{36} \zeta_{4}-\frac{39838}{9} \zeta_{2} \zeta_{3}\right. \\
& \left.-\frac{50680}{9} \zeta_{5}-988 \zeta_{3}^{2}+\frac{12467}{6} \zeta_{6}+496 \zeta_{2} \zeta_{5}+688 \zeta_{3} \zeta_{4}+2260 \zeta_{7}\right) \\
+ & C_{F} n_{c}^{2} n_{f}\left(\frac{83655179}{11664}-\frac{5160215}{972} \zeta_{2}-\frac{639191}{162} \zeta_{3}+\frac{24856}{9} \zeta_{4}+\frac{8624}{9} \zeta_{2} \zeta_{3}\right. \\
& \left.+200 \zeta_{5}-32 \zeta_{3}^{2}-\frac{1201}{3} \zeta_{6}\right)+C_{F} n_{f}^{3}\left(\frac{50558}{2187}+\frac{80}{81} \zeta_{3}-\frac{1880}{81} \zeta_{2}+\frac{40}{9} \zeta_{4}\right) \\
+ & C_{F} n_{c} n_{f}^{2}\left(-\frac{5070943}{5832}+\frac{160903}{243} \zeta_{2}+\frac{14618}{81} \zeta_{3}-\frac{2110}{9} \zeta_{4}-\frac{400}{9} \zeta_{2} \zeta_{3}+\frac{904}{9} \zeta_{5}\right) .
\end{aligned}
$$




\section{Numerical implications}

The lower-order coefficients $B_{l}^{\text {DIS }}$ have the same structure as (2.7), i.e., they contain $-f_{l}^{\mathrm{q}}-B_{l}^{\mathrm{q}}$ and lower-order coefficients. Therefore, by comparing the exact results to an approximation at $\mathrm{N}^{l} \mathrm{LL}$ in which the $\mathrm{L} n_{c}$ expression for $-f_{l}^{\mathrm{q}}-B_{l}^{\mathrm{q}}$ is used together with the exact lower-order coefficients, we can check whether (2.7) with the $\mathrm{L} n_{c}$ results for $f_{4}^{\mathrm{q}}$ and $B_{4}^{\mathrm{q}}$ can be expected to provide a good approximation for $B_{4}^{\text {DIS }}$ and hence $G^{N}$ at the $\mathrm{N}^{4} L L$ accuracy of full QCD.

This comparison is carried out in Fig. 1 for $l=2$ and $l=3$ (at $l=1$ there is no difference between the $\mathrm{L} n_{c}$ limit and full QCD). The $\mathrm{L} n_{c}$ curves are off by less than $0.5 \%$ at $\mathrm{N}^{2} \mathrm{LL}$ and $0.25 \%$ at $\mathrm{N}^{3} L L$ for $G^{\mathrm{DIS}}$ in the $N$-range shown and, at $x \leq 0.9$, for the convolution of its exponential with a schematic but sufficiently realistic form for a quark PDF. Therefore we can safely expect that the $\mathrm{L} n_{c}$ numbers will deviate from (presumably exceed) the exact QCD results by well below $1 \%$.
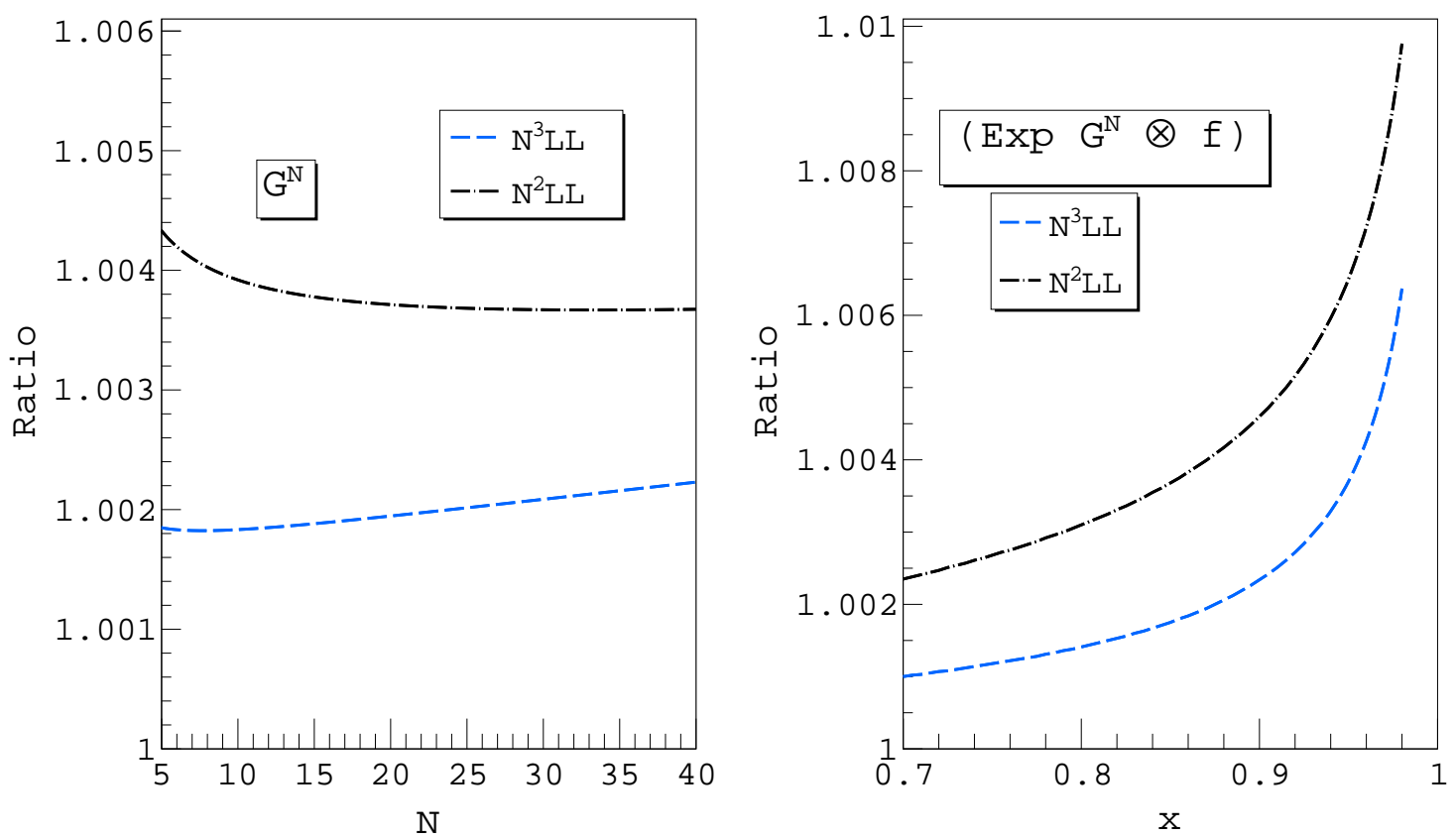

Figure 1: The ratio of the large- $n_{c}$ approximation, defined as above, and the exact results at $\mathrm{N}^{2} \mathrm{LL}$ and $\mathrm{N}^{3} \mathrm{LL}$ for the DIS resummation exponent $G^{N}$ (left) and for the convolution of the exponential with the schematic quark PDF shape $x f=x^{0.5}(1-x)^{3}$ (right) for $\alpha_{\mathrm{s}}=0.2$ and $n_{f}=3$ flavours.

The cumulative effect, relative to the NLL results, of the exact $N^{2} L L$ and $N^{3} L L$ contributions and our new $\mathrm{N}^{4} \mathrm{LL}$ corrections, as above determined using the $\mathrm{L} n_{c}$ limit of $-f_{l}^{\mathrm{q}}-B_{l}^{\mathrm{q}}$ in (2.7), is illustrated in Fig. 2. Unlike the $\mathrm{N}^{3} \mathrm{LL}$ contribution, the $\mathrm{N}^{4} \mathrm{LL}$ correction is almost negligible at $N \leq 15$ and $x \leq 0.9$. Even at $N=40$, the functions $g^{(n)}(\lambda)$ add only $6 \%, 1.6 \%$ and $1 \%$ to the NLL result, respectively, for $n=2, n=3$ and $n=4$, where the latter $\mathrm{L} n_{c}$ result is presumably a slight overestimate. The corresponding $\mathrm{N}^{2} \mathrm{LL}, \mathrm{N}^{3} \mathrm{LL}$ and $\mathrm{N}^{4} \mathrm{LL}$ percentages for the convolution of $\exp G^{N}$ with $x f=x^{0.5}(1-x)^{3}$ at $x=0.95 \mathrm{read} 9.5 \%, 1.5 \%$ and $0.5 \%$, where we have performed the Mellin inversion using a standard contour, see, e.g., [22], which constitutes a 'minimal prescription' contour [4] in the context of the present exponentiation. It appears that the expansion of $G^{N}$ to $\mathrm{N}^{4} \mathrm{LL}$ for the structure functions in inclusive DIS is sufficient for all practical purposes. 

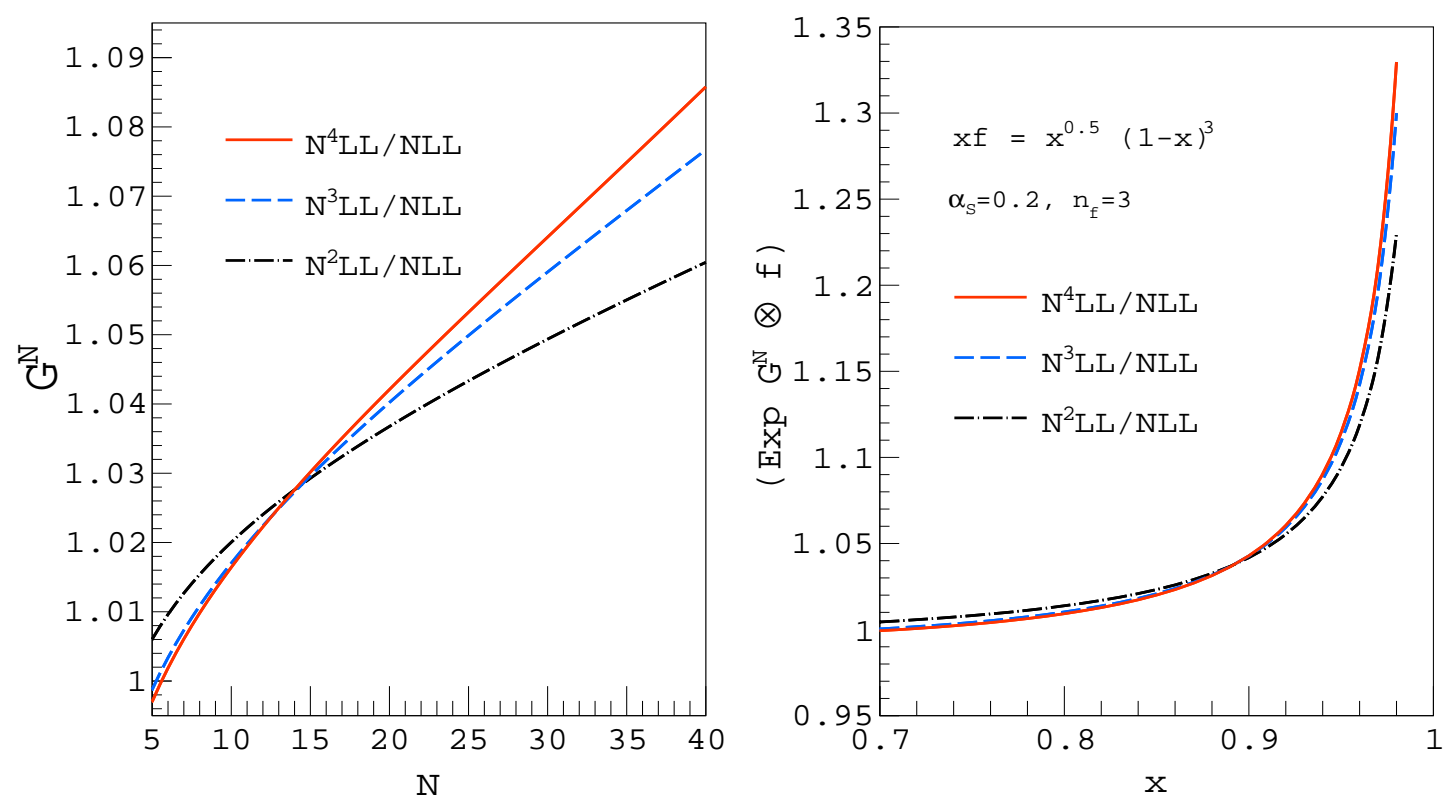

Figure 2: Left: The DIS resummation exponent $G^{N}$ in (2.3) up to $\mathrm{N}^{4} L L$ accuracy, normalized to the NLL result at the standard reference point $\alpha_{\mathrm{s}}=0.2$ for $n_{f}=3$. Right: corresponding $x$-space results for $\exp G^{N}$ after convoluted with a schematic form of a quark PDF of the proton.

\section{Summary and outlook}

We have studied the soft-gluon exponentiation (SGE) of inclusive DIS at the fifth logarithmic $\left(\mathrm{N}^{4} \mathrm{LL}\right)$ order. Recent four-loop results on splitting functions and the quark form factor $[14,15]$ facilitate the exact determination of the form-factor coefficient $f^{\mathrm{q}}$ and the SGE coefficient $B^{\text {DIS }}$ at order $\alpha_{\mathrm{s}}^{4}$ in the large- $n_{c}\left(\mathrm{~L} n_{c}\right)$ limit. Both coefficients are relevant beyond the context of DIS: Like the lightlike quark and gluon cusp anomalous dimensions $A^{\mathrm{q}, \mathrm{g}}$ [10], the quantities $f^{\mathrm{q}, \mathrm{g}}$ are maximally non-Abelian and related by a simple Casimir scaling up to three loops. We expect that the generalized Casimir scaling of [16] also applies to $f^{\mathrm{q}, \mathrm{g}}$, hence our result (2.6) fixes also $f^{\mathrm{g}}$ at large $n_{c}$. The coefficient here called $B^{\text {DIS }}$ is due to the outgoing unobserved quark; hence it contributes to the SGE for many other processes including, e.g., direct photon production [6].

The $\mathrm{L} n_{c}$ approximation to the $\mathrm{N}^{4} \mathrm{LL}$ resummation exponent $G^{N}$ for inclusive DIS, defined as discussed above, is sufficiently accurate to demonstrate that the $\mathrm{N}^{4} \mathrm{LL}$ corrections are small: they contribute well below $1 \%$ over a wide range in $N$ and $x$. As shown in [23], the $1 / N \ln ^{\ell} N$ non-SGE contributions are larger; the highest four of these logarithms are currently known to all orders [23,24] - recall the parameter $\xi_{\mathrm{DIS}_{4}}$ unspecified in [23] was fixed in [24]. We have considered the case of $n_{f}=3$ light flavours. In electromagnetic and neutral-current DIS, also charm production close to threshold needs to be taken into account beyond the threshold for $c \bar{c}$ production, see [25].

\section{References}

[1] A. Accardi et al., Eur. Phys. J. C76 (2016) 471, arXiv:1603.08906.

[2] P. Bolzoni, F. Maltoni, S. Moch and M. Zaro, Phys. Rev. Lett. 105 (2010) 011801, arXiv:1003.4451, M. Cacciari et al., Phys. Rev. Lett. 115 (2015) 082002, arXiv:1506.02660, 
F.A. Dreyer and A. Karlberg, Phys. Rev. Lett. 117 (2016) 072001, arXiv:1606.00840,

J. Currie et al., JHEP 05 (2018) 209, 1803.09973.

[3] J.A.M. Vermaseren, A. Vogt and S. Moch, Nucl. Phys. B724 (2005) 3, hep-ph/0504242, S. Moch, J.A.M. Vermaseren and A. Vogt, Nucl. Phys. B813 (2009) 220, arXiv:0812.4168.

[4] S. Catani, M.L. Mangano, P. Nason and L. Trentadue, Nucl. Phys. B478 (1996) 273, hep-ph/9604351.

[5] H. Contopanagos, E. Laenen and G.F. Sterman, Nucl. Phys. B484 (1997) 303, hep-ph/9604313.

[6] S. Catani, M.L. Mangano and P. Nason, JHEP 07 (1998) 024, hep-ph/9806484.

[7] S. Moch, J.A.M. Vermaseren and A. Vogt, Nucl. Phys. B726 (2005) 317, hep-ph/0506288.

[8] S. Moch and A. Vogt, Phys. Lett. B631 (2005) 48, hep-ph/0508265,

E. Laenen and L. Magnea, Phys. Lett. B632 (2006) 270, hep-ph/0508284.

[9] V. Ravindran, Nucl. Phys. B752 (2006) 173, hep-ph/0603041.

[10] S. Moch, J.A.M. Vermaseren and A. Vogt, Nucl. Phys. B688 (2004) 101, hep-ph/0403192, A. Vogt, S. Moch and J.A.M. Vermaseren, Nucl. Phys. B691 (2004) 129, hep-ph/0404111

[11] S. Moch, J.A.M. Vermaseren and A. Vogt, JHEP 08 (2005) 049, hep-ph/0507039.

[12] S. Moch, J.A.M. Vermaseren and A. Vogt, Phys. Lett. B625 (2005) 245, hep-ph/0508055.

[13] P.A. Baikov et al., Phys. Rev. Lett. 102 (2009) 212002, arXiv:0902.3519,

R.N. Lee, A.V. Smirnov and V.A. Smirnov, JHEP 04 (2010) 020, arXiv:1001.2887,

T. Gehrmann et al., JHEP 06 (2010) 094, arXiv:1004.3653,

T. Gehrmann et al., JHEP 11 (2010) 102, arXiv:1010.4478.

[14] J. Henn et al., JHEP 03 (2017) 139, arXiv:1612.04389.

[15] S. Moch et al., JHEP 10 (2017) 041, arXiv:1707.08315.

[16] S. Moch et al., Phys. Lett. B782 (2018) 627, arXiv:1805.09638.

[17] J.M. Henn, A.V. Smirnov, V.A. Smirnov and M. Steinhauser, JHEP 05 (2016) 066, arXiv:1604.03126, A. Grozin, PoS LL2016 (2016) 053, arXiv:1605.03886,

J. Davies et al., Nucl. Phys. B915 (2017) 335, arXiv:1610.07477,

R.N. Lee, A.V. Smirnov, V.A. Smirnov and M. Steinhauser, Phys. Rev. D96 (2017) 014008, arXiv:1705.06862, A. Grozin, JHEP 06 (2018) 073, arXiv:1805.05050,

R.N. Lee, A.V. Smirnov, V.A. Smirnov and M. Steinhauser, JHEP 02 (2019) 172, arXiv:1901.02898, J.M. Henn, T. Peraro, M. Stahlhofen and P. Wasser, (2019), arXiv:1901.03693.

[18] S. Moch, J.A.M. Vermaseren and A. Vogt, Nucl. Phys. B646 (2002) 181, hep-ph/0209100,

S. Forte and G. Ridolfi, Nucl. Phys. B650 (2003) 229, hep-ph/0209154,

E. Gardi and R.G. Roberts, Nucl. Phys. B653 (2003) 227, hep-ph/0210429.

[19] A. Vogt, Phys. Lett. B497 (2001) 228, hep-ph/0010146,

S. Catani, D. de Florian, M. Grazzini and P. Nason, JHEP 07 (2003) 028, hep-ph/0306211.

[20] G. Das, S. Moch and A. Vogt, DESY 19-088, LTH 1205, to appear.

[21] F. Herzog et al., Phys. Lett. B790 (2019) 436, 1812.11818.

[22] A. Vogt, Comput. Phys. Commun. 170 (2005) 65, hep-ph/0408244.

[23] S. Moch and A. Vogt, JHEP 11 (2009) 099, arXiv:0909.2124.

[24] G.Grunberg, Phys. Lett. B687 (2010) 405, arXiv:0911.4471v5,

A.A. Almasy, G. Soar and A. Vogt, JHEP 03 (2011) 030, arXiv:1012.3352.

[25] H. Kawamura, N.A. Lo Presti, S. Moch and A. Vogt, Nucl. Phys. B864 (2012) 399, arXiv:1205.5727 